\title{
Increased mortality and ICD therapies in ischemic versus non-ischemic dilated cardiomyopathy patients with cardiac resynchronization having survived until first device replacement
}

Thomas Beiert, Swanda Straesser, Robert Malotki, Florian Stöckigt, Jan W. Schrickel, René P. Andrié

Department of Internal Medicine II, University Hospital Bonn, Rheinische FriedrichWilhelms University, Bonn, Germany

Submitted: 8 March 2017

Accepted: 5 May 2017

Arch Med Sci 2019; 15 (4): 845-856

DOI: https://doi.org/10.5114/aoms.2018.75139

Copyright @ 2018 Termedia \& Banach

\section{Abstract}

Introduction: Cardiac resynchronization therapy combined with an implantable cardioverter defibrillator (CRT-D) is widely applied in heart failure patients. Sufficient data on arrhythmia and defibrillator therapies during long-term follow-up of more than 4 years are lacking and data on mortality are conflicting. We aimed to characterize the occurrence of ventricular arrhythmia, respective defibrillator therapies and mortality for several years following CRT-D implantation or upgrade.

Material and methods: Eighty-eight patients with ischemic (ICM) or non-ischemic dilated cardiomyopathy (DCM) and at least one CRT-D replacement were included in this study and analyzed for incidence of non-sustained ventricular tachycardia (NSVT), defibrillator shocks, anti-tachycardia pacing (ATP) and mortality.

Results: ICM was the underlying disease in $59 \%$, DCM in $41 \%$ of patients. During a mean follow-up of $76.4 \pm 24.8$ months the incidence of appropriate defibrillator therapies (shock or ATP) was $46.6 \%$ and was elevated in ICM compared to DCM patients $(57.7 \%$ vs. $30.6 \%$, respectively; $p=0.017)$. Kaplan-Meier analysis revealed significantly higher ICD therapy-free survival rates in DCM patients $(p=0.031)$. Left ventricular ejection fraction, NSVT per year and ICM (vs. DCM) were independent predictors of device intervention. The ICM patients showed increased mortality compared to DCM patients, with cumulative all-cause mortality at 9 years of follow-up of $45.4 \%$ and $10.6 \%$, respectively. Chronic renal failure, peripheral artery disease and chronic obstructive pulmonary disease were independent predictors of mortality.

Conclusions: The clinical course of patients with ICM and DCM treated with CRT-D differs significantly during long-term follow-up, with increased mortality and incidence of ICD therapies in ICM patients.

Key words: cardiac resynchronization therapy, implantable cardioverter defibrillator, heart failure, ischemic cardiomyopathy, non-ischemic dilated cardiomyopathy.

\section{Introduction}

Cardiac resynchronization therapy (CRT) has evolved into a broadly used treatment for patients suffering from chronic heart failure with severely reduced left ventricular ejection fraction (LVEF) and left bundle

\author{
Corresponding author: \\ Dr. Thomas Beiert \\ Department of Internal \\ Medicine II \\ University Hospital Bonn \\ Sigmund-Freud Str. 25 \\ 53127 Bonn, Germany \\ Phone: +49 22828715507 \\ Fax: +49 22828711057 \\ E-mail: Thomas.Beiert@ \\ ukbonn.de
}


branch block to reduce morbidity and mortality $[1,2]$. Furthermore, it is known that the risk of sudden cardiac death $(\mathrm{SCD})$ due to ventricular tachycardia (VT) or ventricular fibrillation (VF) can be reduced by an implantable cardioverter defibrillator (ICD) in these patients [2, 3]. Up to now there is insufficient clinical data from randomized clinical trials to prove superiority of a combined CRT and ICD implantation (CRT-D) over CRT pacemakers (CRT-P) [1, 4]. Besides improvement of LVEF there is evidence for anti-arrhythmic effects following CRT implantation [5]. A recent meta-analysis of more than 12,000 patients demonstrated a reduction in the risk of all-cause mortality for CRT-D, which was more pronounced in patients suffering from ischemic cardiomyopathy (ICM) compared to non-ischemic dilated cardiomyopathy (DCM) [4]. Detailed data on arrhythmic events and respective ICD therapies in CRT-D patients during long-term follow-up are scarce. Analysis of the German DEVICE-registry did neither show a mortality difference nor a disparity in first ICD shock delivery according to heart failure etiology in CRT-D patients [6]. McLeod et al. observed no significant difference in appropriate or inappropriate shocks but reported a greater improvement of left ventricular systolic function (also shown by Barsheshet et al. [7] and in the MADIT-CRT study [8]) and a significant difference in survival favoring DCM patients [9]. In ICD patients as well, most studies reported similar shock delivery rates for ICM and DCM $[10,11]$.

Follow-up in the above-mentioned studies rarely exceeds 4 years and we therefore aimed to delineate differences between ICM and DCM patients treated with CRT-D in incidence of ventricular arrhythmia and ICD therapies as well as mortality during long-term follow-up of at least 5 years.

\section{Material and methods}

\section{Study design}

The present study is a retrospective single-center study at a German tertiary care university hospital. The study was approved by the local ethics committee. All procedures performed were in accordance with the ethical standards of the institutional research committee and with the $1964 \mathrm{Hel}$ sinki declaration. To ensure long-term follow-up of more than 5 years, only patients with CRT-D replacement due to end of the battery lifespan between February 2007 and November 2015 were included. This selection resulted in 88 patients with first CRT-D implantation between 2002 and 2011 that were routinely (3-6 months intervals) followed up as outpatients at our department.

CRT-D devices were either implanted primarily or patients were upgraded from pre-existing pacemaker, ICD or CRT-P devices. Both directly implanted and upgraded patients were only included following at least one replacement of CRT-D due to end of the battery lifespan.

The primary endpoints were mortality and a composite of appropriate ATP or shock by ICD. The secondary outcomes were appropriate ATP or shock by ICD, respectively.

The LVEF was calculated via transthoracic echocardiography by biplane Simpson method. Change in LVEF was defined as difference between the LVEF prior to CRT-D implantation/upgrade and the last documented LVEF. To account for differences due to varying follow-up durations the change in LVEF, total numbers of shocks for VT/VF, ATP for VT/VF and non-sustained VT/VF (NSVT/NSVF) are presented per year.

\section{Arrhythmia events and device therapies}

Each individual follow-up at our center was analyzed for occurrence of ventricular arrhythmia and ICD therapies (shock or ATP) starting with the first visit after initial CRT-D implantation or upgrade. In patients having received an upgrade from ICD to CRT-D, arrhythmia and device therapies before the upgrade were not included. All arrhythmia episodes and device therapies were analyzed by two different physicians with long-standing experience in device therapy. If in doubt, electrograms were sent to the respective company. Appropriate therapy was defined as shock or ATP for real VT or VF following analysis of the intracardiac electrograms. NSVT and NSVF were defined as true arrhythmia episodes $<30 \mathrm{~s}$ in the respective programmed $\mathrm{VT}$ or VF zones.

\section{Device programming}

CRT-D devices were programmed individually according to standard clinical care at our center. The pre-set parameters were almost never used. In general, two or three therapy zones (mainly one VT zone, one VF zone and possibly an additional fast VT (FVT) zone) were programmed. VT was primarily treated with ATP and possibly consecutive ICD shocks. VF was primarily treated with ICD shock with ATP during charging if available.

Typically, defibrillator settings (for primary prevention) were programmed as follows: VT zone: cycle length: 350-400 ms; detection: 20-24; redetection: 12; VF zone: cycle length: 300-320 ms; detection: 18 of 24; re-detection: 12 of 16 .

After emergence of the MADIT-RIT study [12] ICD programming was adapted to: VT zone: cycle length: 330-400 ms; detection: 24-28; re-detection: 12; VF zone: cycle length: 270-315 ms; detection: 30 of 40; re-detection: 12 of 16 . 


\section{Statistical analysis}

Continuous variables were tested for normal distribution using the Kolmogorov-Smirnov and Shapiro-Wilk tests. Normally distributed continuous variables are expressed as mean \pm standard deviation (SD). Non-normally distributed continuous variables are shown as median with interquartile range $\left(25^{\text {th }}-75^{\text {th }}\right.$ percentile). The unpaired two-tailed Student's t-test or one-way ANOVA with Tukey post-hoc test were performed for normally distributed continuous variables, whereas the Mann-Whitney $U$ test was chosen for non-normally distributed continuous variables. The homogeneity of variance was assessed using the Levene test. Categorical variables are displayed as frequencies and percentages and the $\chi^{2}$ test was used for data analysis. The cumulative survival plots (free of appropriate therapy/ shock/ATP; survival) were estimated according to the Kaplan-Meier method. Survival in groups was compared with the log rank test. Univariate Cox regression analysis was performed to identify significant independent predictors of outcome. For multivariate analysis (backward stepwise selection method) significant predictors from the univariate analysis were included. Results are reported as the adjusted hazard ratio $(H R)$ with 95\% confidence interval (Cl). A two-sided $p$-value $<0.05$ was considered statistically significant. Statistical analyses were performed using SPSS software version 23.0 (SPSS, Chicago, IL).

\section{Results}

\section{Study population}

The study population consisted of 88 patients who received a CRT-D according to guideline recommendations for the treatment of chronic heart failure as either de novo implantation or an upgrade from a previously implanted ICD, pacemaker or CRT-P. Mean follow-up was $76.4 \pm 24.8$ months. ICM was the underlying etiology in 52 (59\%) patients and DCM in 36 (41\%) patients. The mean age of our study population at first CRT-D implantation/upgrade was $68.0 \pm 9.1$ years and the mean LVEF prior to CRT-D was $25 \pm 7 \%$. Baseline characteristics and medication of all patients and the ICM and DCM subgroups are presented in Table I. ICM patients were older than DCM patients $(p=$ $0.011)$ and more often male $(p=0.032)$. DCM patients had a higher body mass index $(p=0.009)$. Of note, there was no difference in BMI between patients without sleep apnea and those with central or obstructive sleep apnea $\left(27.0 \pm 5.1 \mathrm{~kg} / \mathrm{m}^{2}\right.$, $27.4 \pm 4.1 \mathrm{~kg} / \mathrm{m}^{2}$ and $28.9 \pm 4.1 \mathrm{~kg} / \mathrm{m}^{2}$, respectively). The use of statins was more frequent in ICM patients $(p=0.002)$. $76.1 \%$ of the whole study population were treated with an ACE inhibitor or AT-1 blocker, $92.0 \%$ received a $\beta$-blocker and $45.5 \%$ were additionally treated with an aldosterone antagonist. Functional NYHA class was only numerically higher in ICM patients $(p=0.095)$.

\section{Device-related characteristics}

The majority of devices (89.8\%) were implanted for primary prevention of SCD. The mean time to first CRT-D replacement due to end of the battery lifespan was $45.6 \pm 10.3$ months and to second replacement $101.1 \pm 14.3$ months. There was no significant difference between ICM and DCM patients. Atrial pacing (AP) and bi-ventricular pacing (biVP) rates at last follow-up were comparable between the groups. Both ICM and DCM patients had excellent biVP rates with a median of $99 \%$ each. The median change in LVEF per year was $0.4 \%(0.0,1.5)$ for ICM and $1.5 \%(0.0,3.3)$ for DCM patients $(p=0.112)$. All essential device-related characteristics are shown in Table II.

\section{ICD therapies in ICM and DCM patients}

Appropriate shocks were delivered in 29 (33.0\%) and ATP in 35 (39.8\%) patients. Forty-one (46.6\%) patients had any device therapy (appropriate shock or ATP), with significantly higher therapy rates in patients with ICM (57.7\%) compared to DCM patients $(30.6 \%)(p=0.017)$. A detailed overview of ICD therapies is displayed in Table II. A total of 6 (6.8\%) patients received inappropriate shocks, which did not differ between the groups $(p=0.221)$. ICM patients had significantly more ATP for VT treatment per year $(p=0.049)$. There was no difference in the incidence or amount of NSVT/NSVF between the groups. The secondary endpoints, shock-free (Figure 1 A) and ATP-free (Figure 1 B) survival, were only numerically higher in DCM patients. For the combined primary endpoint, DCM patients had a significantly higher therapy-free survival compared to ICM patients (Figure $1 \mathrm{C}$ ). The cumulative incidence of ICD therapies at 3, 6 and 9 years of follow-up was $42.3 \%$, $58.2 \%$ and $62.0 \%$ for ICM patients and $25.0 \%$, $29.8 \%$ and $29.8 \%$ for DCM patients, respectively. Time to first therapy was numerically longer in DCM patients (ICM: $49.3 \pm 32.2$ months, DCM: $61.4 \pm 37.0$ months; $p=0.107)$.

\section{Predictors of ICD intervention}

Univariate Cox proportional analyses for appropriate device intervention revealed a significant influence of male gender, LVEF (per 5\%), ICM (versus DCM) and NSVT (Table III). Subsequently these were fitted as independent variables in a multivariate Cox proportional hazards model with ICD therapy as the dependent variable. In this model, ICM patients had a $150 \%$ higher risk for defibril- 
Table I. Baseline characteristics of study population

\begin{tabular}{|c|c|c|c|c|}
\hline Parameter & All patients $(n=88)$ & $\operatorname{ICM}(n=52)$ & $\operatorname{DCM}(n=36)$ & $P$-value \\
\hline Age at $1^{\text {st }}$ CRT-D [years] & $68.0 \pm 9.1$ & $70.0 \pm 8.2$ & $65.1 \pm 9.6$ & 0.011 \\
\hline $\operatorname{LVEF}(\%)$ & $25 \pm 7$ & $25 \pm 6$ & $26 \pm 7$ & 0.197 \\
\hline Male (\%) & $71(80.7)$ & $46(88.5)$ & $25(69.4)$ & 0.032 \\
\hline Diabetes (\%) & $33(37.5)$ & $20(38.5)$ & $13(36.1)$ & 1.000 \\
\hline Hypertension (\%) & $62(70.5)$ & $41(78.8)$ & $21(58.3)$ & 0.057 \\
\hline Dyslipidemia (\%) & $29(33.0)$ & $21(40.4)$ & $8(22.2)$ & 0.106 \\
\hline BMI $\left[\mathrm{kg} / \mathrm{m}^{2}\right]$ & $27.4 \pm 4.8$ & $26.2 \pm 4.5$ & $29.0 \pm 4.8$ & 0.009 \\
\hline Obesity (\%) & $28(33.7)$ & $13(26.5)$ & $15(44.1)$ & 0.106 \\
\hline Peripheral artery disease (\%) & $16(17)$ & $12(23.1)$ & $3(8.3)$ & 0.088 \\
\hline Coronary artery disease (\%) & $55(62.5)$ & $49(94.2)$ & $6(16.7)$ & $<0.0001$ \\
\hline Previous MI (\%) & $29(33.0)$ & $27(51.9)$ & $2(5.6)$ & $<0.0001$ \\
\hline Previous CABG (\%) & $15(17.0)$ & $15(28.8)$ & $0(0.0)$ & $<0.0001$ \\
\hline Previous myocarditis (\%) & $7(8.0)$ & $2(3.8)$ & $5(13.9)$ & 0.117 \\
\hline Previous CPR (\%) & $10(11.4)$ & $8(15.4)$ & $2(5.6)$ & 0.189 \\
\hline Previous stroke (\%) & $8(9.1)$ & $7(13.5)$ & $1(2.8)$ & 0.134 \\
\hline Chronic renal failure (\%) & $39(44.3)$ & $27(51.9)$ & $12(33.3)$ & 0.126 \\
\hline COPD (\%) & $16(18.2)$ & $12(23.1)$ & $4(11.1)$ & 0.173 \\
\hline Obstructive sleep apnea (\%) & $14(16.3)$ & $8(15.7)$ & $6(17.1)$ & 1.000 \\
\hline Central sleep apnea (\%) & $10(11.6)$ & $8(15.7)$ & $2(5.7)$ & 0.190 \\
\hline Paroxysmal AF (\%) & $21(23.9)$ & $12(23.1)$ & $9(25.0)$ & 1.00 \\
\hline Persistent AF (\%) & $18(20.5)$ & $14(26.9)$ & $4(11.1)$ & 0.106 \\
\hline Permanent AF (\%) & $16(18.2)$ & $9(17.3)$ & $7(19.4)$ & 0.787 \\
\hline \multicolumn{5}{|l|}{ Medication at last follow-up: } \\
\hline ACE inhibitor/AT-1 blocker (\%) & $67(76.1)$ & $37(71.2)$ & $30(83.3)$ & 0.214 \\
\hline Aldosterone antagonist (\%) & $40(45.5)$ & $25(48.1)$ & $15(41.7)$ & 0.664 \\
\hline$\beta$-Blocker (\%) & $81(92.0)$ & $48(92.3)$ & $33(91.7)$ & 1.00 \\
\hline Diuretic (\%) & $81(92.0)$ & $48(92.3)$ & $33(91.7)$ & 1.00 \\
\hline Acetylsalicylic acid (\%) & $41(46.6)$ & $28(53.8)$ & $13(36.1)$ & 0.130 \\
\hline Statin (\%) & $67(76.1)$ & $46(88.5)$ & $21(58.3)$ & 0.002 \\
\hline Digoxin/digitoxin (\%) & $23(26.1)$ & $15(28.8)$ & $8(22.2)$ & 0.623 \\
\hline Amiodarone (\%) & $31(35.2)$ & $20(38.5)$ & $11(30.6)$ & 0.501 \\
\hline Phenprocoumon (\%) & $45(51.1)$ & $31(59.6)$ & $14(38.9)$ & 0.082 \\
\hline Direct oral anticoagulant (\%) & $11(12.5)$ & $6(11.5)$ & $5(13.9)$ & 0.754 \\
\hline NYHA at last follow-up & $3.0(2.0,3.0)$ & $3.0(2.0,3.0)$ & $2.0(2.0,3.0)$ & 0.095 \\
\hline
\end{tabular}

Values are $n(\%)$, mean \pm standard deviation or median $\left(25^{\text {th }}-75^{\text {th }}\right.$ percentile). ICM - ischemic cardiomyopathy, DCM - non-ischemic dilated cardiomyopathy, LVEF - left ventricular ejection fraction, BMI - body mass index. Obesity was defined as BMI $\geq 30 \mathrm{~kg} / \mathrm{m}^{2}$. MI - myocardial infarction, $C A B G$ - coronary artery bypass grafting, CPR - cardiopulmonary resuscitation, COPD - chronic obstructive pulmonary disease, $A F-$ atrial fibrillation. 
Increased mortality and ICD therapies in ischemic versus non-ischemic dilated cardiomyopathy patients with cardiac resynchronization having survived until first device replacement

Table II. Device-related characteristics of study population

\begin{tabular}{|c|c|c|c|c|}
\hline Parameter & All patients $(n=88)$ & ICM $(n=52)$ & $\operatorname{DCM}(n=36)$ & $P$-value \\
\hline Upgrade to CRT-D (\%): & $25(28.4)$ & $21(40.4)$ & $4(11.1)$ & 0.004 \\
\hline Upgrade from ICD & $12(13.6)$ & $11(21.2)$ & $1(2.8)$ & \\
\hline Upgrade from CRT-P & $2(2.3)$ & $2(3.8)$ & $0(0.0)$ & \\
\hline Upgrade from PM & $11(12.5)$ & $8(15.4)$ & $3(8.3)$ & \\
\hline $\begin{array}{l}\text { Time to } 1^{\text {st }} \text { CRT-D replacement } \\
\text { [months] }\end{array}$ & $45.6 \pm 10.3$ & $49.8 \pm 8.8$ & $49.2 \pm 12.1$ & 0.813 \\
\hline $\begin{array}{l}\text { Time to } 2^{\text {nd }} \text { CRT-D replacement } \\
\text { [months] }\end{array}$ & $101.1 \pm 14.3$ & $109.0 \pm 18.2$ & $97.2 \pm 11.0$ & 0.137 \\
\hline Follow-up [months] & $76.4 \pm 24.8$ & $73.7 \pm 23.7$ & $80.2 \pm 26.1$ & 0.228 \\
\hline Primary prevention (\%) & $79(89.8)$ & $46(88.5)$ & $33(91.7)$ & 0.732 \\
\hline $\mathrm{AP}$ rate at last FU [\%] & $11.5(0.3,75.7)$ & $13.0(0.3,70.0)$ & $6.8(0.2,75.7)$ & 0.715 \\
\hline biVP rate at last FU [\%] & $99.0(97.8,99.7)$ & $99.0(97.5,99.6)$ & $99.0(98.2,99.7)$ & 0.341 \\
\hline Change in LVEF per year [\%] & $0.7(0.0,2.6)$ & $0.4(0.0,1.5)$ & $1.5(0.0,3.3)$ & 0.112 \\
\hline Appropriate shock (\%) & $29(33.0)$ & $21(40.4)$ & $8(22.2)$ & 0.106 \\
\hline Appropriate shock for VT (\%) & $23(26.1)$ & $16(30.8)$ & $7(19.4)$ & 0.325 \\
\hline Appropriate shock for VF (\%) & $18(20.5)$ & $14(26.9)$ & $4(11.1)$ & 0.106 \\
\hline Inappropriate shock (\%) & $6(6.8)$ & $2(3.8)$ & $4(11.1)$ & 0.221 \\
\hline ATP in total (\%) & $36(40.9)$ & $26(50.0)$ & $10(27.8)$ & 0.048 \\
\hline ATP for VT (\%) & $36(40.9)$ & $26(50.0)$ & $10(27.8)$ & 0.048 \\
\hline ATP for VF (\%) & $9(10.2)$ & $5(9.6)$ & $4(11.1)$ & 1.000 \\
\hline Appropriate shock or ATP (\%) & $41(46.6)$ & $30(57.7)$ & $11(30.6)$ & 0.017 \\
\hline NSVT (\%) & $68(77.3)$ & $37(71.2)$ & $31(86.1)$ & 0.125 \\
\hline NSVF (\%) & $3(3.4)$ & $2(5.6)$ & $1(1.9)$ & 0.565 \\
\hline Shocks for VT per year & $0.23 \pm 0.62$ & $0.22 \pm 0.49$ & $0.23 \pm 0.78$ & 0.483 \\
\hline Shocks for VF per year & $0.16 \pm 0.61$ & $0.18 \pm 0.72$ & $0.14 \pm 0.42$ & 0.309 \\
\hline ATP for VT per year & $2.83 \pm 14.64$ & $4.34 \pm 18.90$ & $0.65 \pm 1.96$ & 0.049 \\
\hline ATP for VF per year & $0.53 \pm 3.88$ & $0.73 \pm 5.01$ & $0.24 \pm 0.82$ & 0.771 \\
\hline NSVT per year & $4.47 \pm 7.95$ & $3.96 \pm 6.43$ & $5.21 \pm 9.78$ & 0.396 \\
\hline NSVF per year & $0.02 \pm 0.14$ & $0.003 \pm 0.02$ & $0.04 \pm 0.22$ & 0.345 \\
\hline
\end{tabular}

Values are $n(\%)$, mean \pm standard deviation or median $\left(25^{\text {th }}-75^{\text {th }}\right.$ percentile). ICM - ischemic cardiomyopathy, DCM - non-ischemic dilated cardiomyopathy, PM - pacemaker, FU - follow-up, AP - atrial pacing rate, biVP - bi-ventricular pacing rate, LVEF - left ventricular ejection fraction, VT - ventricular tachycardia, VF-ventricular fibrillation, ATP - anti-tachycardia pacing, NSVT/NSVF-non-sUstained ventricular tachycardia/fibrillation.

lator therapies when compared to DCM patients $(H R=2.529)$. Each NSVT per year resulted in a $6 \%$ increase in the risk for ICD intervention $(\mathrm{HR}=$ 1.056), whereas each $5 \%$ of LVEF reduced the risk for ICD therapy by $30 \%(H R=0.703)$ (Table III).

\section{Mortality in CRT-D patients}

DCM patients had a significantly higher survival rate compared to ICM patients (Figure 2). The $\mathrm{Cu}$ mulative all-cause mortality at 3, 6 and 9 years of follow-up was $0.0 \%, 12.5 \%$ and $45.4 \%$ for ICM and $0.0 \%, 6.8 \%$ and $10.6 \%$ for DCM patients, respectively. Since only patients with at least one CRT-D replacement were included in this study, there were no deaths $<50$ months after implantation.

\section{Predictors of mortality}

Univariate Cox proportional analyses for mortality revealed a significant influence of central sleep apnea, ICM (versus DCM), peripheral artery 
A

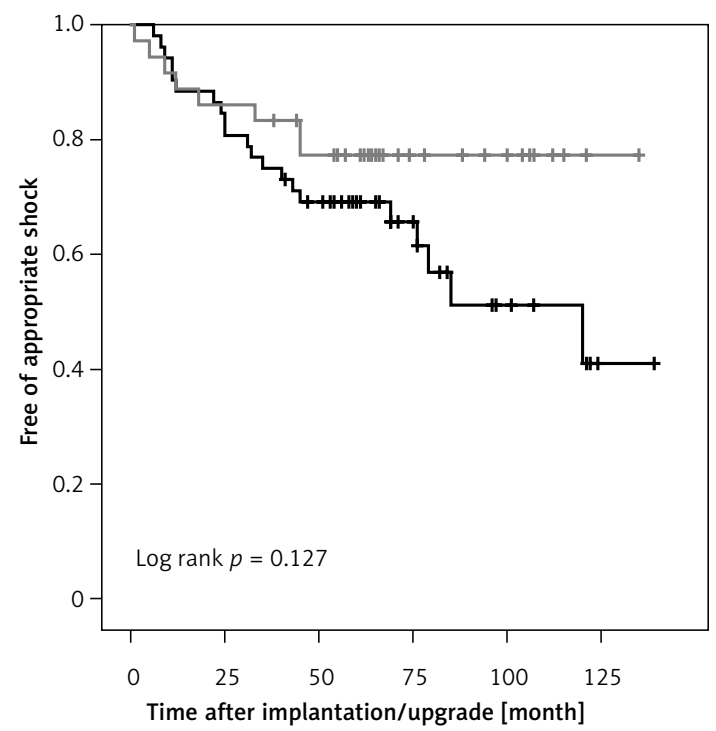

No. at risk

$\begin{array}{llllllllllll}\text { ICM } & 52 & 46 & 44 & 38 & 29 & 21 & 15 & 8 & 6 & 4 & 1 \\ \text { DCM } & 36 & 32 & 31 & 29 & 23 & 15 & 11 & 9 & 6 & 2 & 1\end{array}$

ПICM $\neg D C M$

C

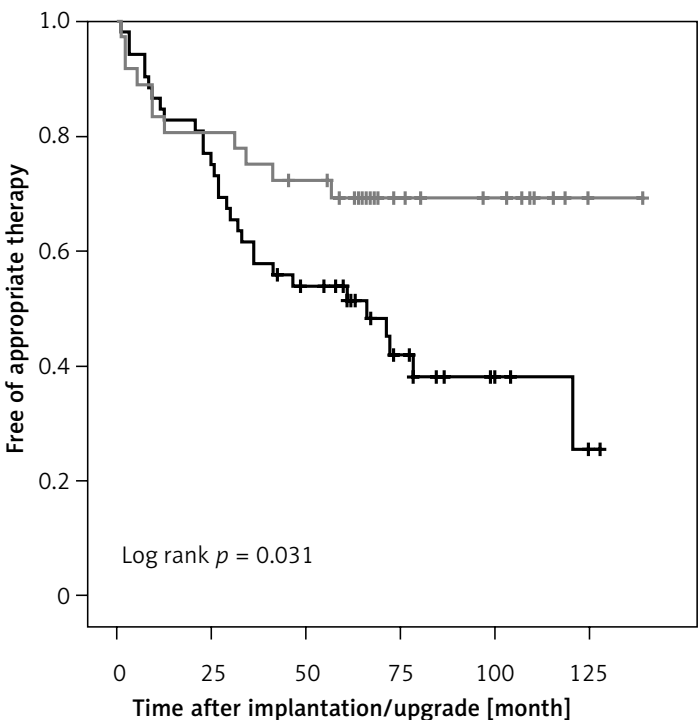

No. at risk

$\begin{array}{llllllllllll}\text { ICM } & 52 & 43 & 39 & 29 & 22 & 16 & 10 & 5 & 4 & 2 & 0 \\ \text { DCM } & 36 & 29 & 29 & 27 & 22 & 14 & 10 & 9 & 6 & 2 & 1\end{array}$

disease, chronic renal failure and chronic obstructive pulmonary disease (COPD) (Table IV). Subsequently these were fitted as independent variables in a multivariate Cox proportional hazards model with mortality as the dependent variable. In this model, patients suffering from peripheral artery disease had a $279 \%$ higher risk for mortality $(\mathrm{HR}=3.786)$, whereas in patients with COPD mortality increased by $289 \%(H R=3.889)$. Chronic renal failure led to a $225 \%$ increase of mortality $(H R=3.236)$ (Table IV).
B

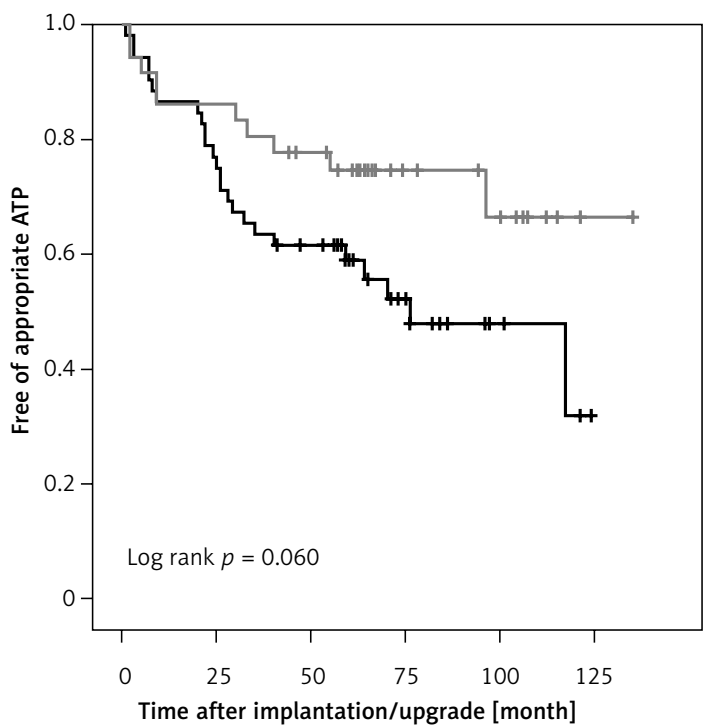

No. at risk

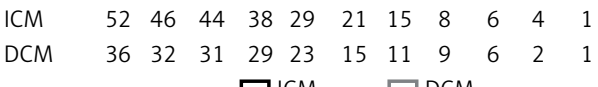

$\neg I C M \quad \curvearrowleft D C M$

Figure 1. ICD therapy-free survival in ICM and DCM patients. A - Kaplan-Meier estimates for survival free of appropriate shock delivered by CRT-D. B - Kaplan-Meier estimates for survival free of appropriate ATP delivered by CRT-D. C - Kaplan-Meier estimates for survival free of appropriate therapy (shock or ATP) delivered by CRT-D

ICM - ischemic cardiomyopathy, DCM - non-ischemic dilated cardiomyopathy, CRT-D-cardiac resynchronization therapy combined with an implantable cardioverter defibrillator (ICD), ATP - anti-tachycardia pacing.

\section{Subgroup of patients with primary preventive CRT-D implantation}

Seventy-nine (89.8\%) patients received their CRT-D for primary prevention. This subgroup of our study population revealed reduced therapy-free survival and increased mortality in ICM compared to DCM patients as well (Figures $3 \mathrm{~A}, \mathrm{~B}$, respectively). ICM, NSVT and LVEF were predictors of appropriate device intervention (Table $\mathrm{V}$ ), whereas peripheral artery disease and COPD predicted mortality (Table VI). 
Increased mortality and ICD therapies in ischemic versus non-ischemic dilated cardiomyopathy patients with cardiac resynchronization having survived until first device replacement

Table III. Univariate and multivariate Cox proportional hazard ratios (HR) for appropriate CRT-D intervention (shock or anti-tachycardia pacing)

\begin{tabular}{|lcccc|}
\hline Parameter & \multicolumn{2}{c}{ Univariate } & \multicolumn{1}{c|}{ Multivariate } \\
\cline { 2 - 5 } & \multicolumn{1}{c}{ HR $(95 \% \mathrm{Cl})$} & P-value & HR (95\% Cl) & P-value \\
\hline Age, per 5 years & $1.068(0.898-1.270)$ & 0.459 & & \\
\hline Male & $3.989(1.229-12.948)$ & 0.021 & $1.778(0.518-6.107)$ & 0.360 \\
\hline ICM & $2.100(1.051-4.197)$ & 0.036 & $2.529(1.201-5.325)$ & 0.015 \\
\hline Diabetes & $0.521(0.261-1.040)$ & 0.064 & & \\
\hline Hypertension & $1.083(0.542-2.163)$ & 0.822 & & \\
\hline BMI, per 1 kg/m ${ }^{2}$ & $0.968(0.904-1.035)$ & 0.342 & & \\
\hline Obesity & $0.565(0.267-1.198)$ & 0.137 & & \\
\hline Dyslipidemia & $1.450(0.778-2.702)$ & 0.242 & & \\
\hline Peripheral artery disease & $1.256(0.579-2.729)$ & 0.564 & & \\
\hline Previous stroke & $1.603(0.627-4.100)$ & 0.325 & & \\
\hline COPD & $1.423(0.679-2.983)$ & 0.350 & & \\
\hline Chronic renal failure & $1.084(0.585-2.010)$ & 0.797 & & \\
\hline Obstructive sleep apnea & $0.489(0.173-1.378)$ & 0.176 & & \\
\hline Central sleep apnea & $0.955(0.338-2.694)$ & 0.930 & & \\
\hline Previous CPR & $0.639(0.197-2.074)$ & 0.456 & & \\
\hline NSVT per year & $1.047(1.018-1.077)$ & 0.001 & \\
\hline LVEF, per 5\% & $0.704(0.562-0.882)$ & 0.002 & & \\
\hline
\end{tabular}

ICM - ischemic cardiomyopathy, BMI - body mass index, COPD - chronic obstructive pulmonary disease, CPR - cardiopulmonary resuscitation, NSVT - non-sustained ventricular tachycardia, LVEF - left ventricular ejection fraction.

\section{Direct implantation or upgrade to CRT-D}

In total, 25 (28.4\%) patients received an upgrade to CRT-D (Table II). Significantly more ICM patients were upgraded compared to DCM patients (40.4\% and $11.1 \%$, respectively; $p=0.004)$, especially when a previous ICD was upgraded (12 patients in total). In 2 patients a CRT-P and in 11 patients a pacemaker was upgraded to CRT-D. ICD therapy-free survival was similar between directly implanted and upgraded CRT-D devices (Figure 4). Comparing directly implanted and upgraded CRT-D in ICM or DCM patients alone, resulted in comparable event-free survival rates as well (data not shown).

\section{Discussion}

The present study evaluated the occurrence of ventricular arrhythmia and concomitant ICD therapies in CRT-D patients who survived until first device replacement due to end of the battery lifespan. This pre-selection was chosen to guarantee long-term real-world follow-up for a median of 6-7 years, since previous studies rarely provided information about more than 3-4 years. Data were analyzed for differences between the two major cardiomyopathy etiologies, ischemic and non-ischemic dilated cardiomyopathy, which cause heart

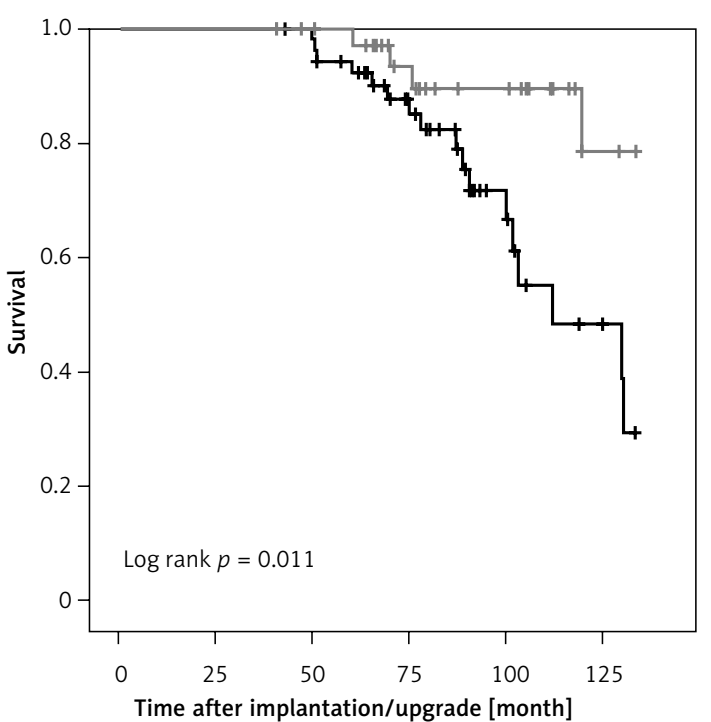

No. at risk

$$
\begin{aligned}
& \begin{array}{llllllllllll}
\text { ICM } & 52 & 52 & 52 & 52 & 50 & 42 & 32 & 21 & 13 & 8 & 4
\end{array} \\
& \begin{array}{llllllllllll}
\text { DCM } & 36 & 36 & 36 & 35 & 34 & 29 & 22 & 18 & 15 & 10 & 3
\end{array} \\
& \text { ПICM ПDCM }
\end{aligned}
$$

Figure 2. Cumulative survival in ICM and DCM patients. Kaplan-Meier estimates for survival in patients treated with cardiac resynchronization therapy combined with an implantable cardioverter defibrillator (CRT-D) according to disease etiology ICM - ischemic cardiomyopathy, DCM - non-ischemic dilated cardiomyopathy. 
Thomas Beiert, Swanda Straesser, Robert Malotki, Florian Stöckigt, Jan W. Schrickel, René P. Andrié

Table IV. Univariate and multivariate Cox proportional hazard ratios (HR) for mortality

\begin{tabular}{|c|c|c|c|c|}
\hline \multirow[t]{2}{*}{ Parameter } & \multicolumn{2}{|l|}{ Univariate } & \multicolumn{2}{|c|}{ Multivariate } \\
\hline & $\mathrm{HR}(95 \% \mathrm{Cl})$ & $P$-value & $\mathrm{HR}(95 \% \mathrm{Cl})$ & $P$-value \\
\hline Age, per 5 years & $1.346(1.000-1.813)$ & 0.050 & & \\
\hline Male & $4.876(0.654-36.370)$ & 0.122 & & \\
\hline ICM & $3.789(1.266-11.343)$ & 0.017 & $1.511(0.421-5.424)$ & 0.527 \\
\hline Diabetes & $0.790(0.305-2.045)$ & 0.627 & & \\
\hline Hypertension & $1.205(0.465-3.124)$ & 0.702 & & \\
\hline $\mathrm{BMI}$, per $1 \mathrm{~kg} / \mathrm{m}^{2}$ & $0.962(0.871-1.063)$ & 0.447 & & \\
\hline Obesity & $0.556(0.202-1.531)$ & 0.256 & & \\
\hline Dyslipidemia & $1.108(0.457-2.686)$ & 0.820 & & \\
\hline Peripheral artery disease & $4.844(1.960-11.969)$ & 0.001 & $3.786(1.531-9.367)$ & 0.004 \\
\hline Previous stroke & $1.798(0.527-6.129)$ & 0.349 & & \\
\hline COPD & 4.067 (1.704-9.711) & 0.002 & $3.889(1.598-9.460)$ & 0.003 \\
\hline Chronic renal failure & $3.164(1.274-7.860)$ & 0.013 & $3.236(1.270-8.243)$ & 0.014 \\
\hline Obstructive sleep apnea & $1.285(0.366-4.503)$ & 0.696 & & \\
\hline Central sleep apnea & $2.927(1.057-8.100)$ & 0.039 & $1.289(0.398-4.180)$ & 0.672 \\
\hline Previous CPR & $2.448(0.703-8.521)$ & 0.159 & & \\
\hline NSVT per year & $0.962(0.890-1.040)$ & 0.331 & & \\
\hline LVEF, per $5 \%$ & $0.868(0.645-1.167)$ & 0.349 & & \\
\hline
\end{tabular}

ICM - ischemic cardiomyopathy, BMI - body mass index, COPD - chronic obstructive pulmonary disease, CPR - cardiopulmonary resuscitation, NSVT - non-sustained ventricular tachycardia, LVEF - left ventricular ejection fraction.

A

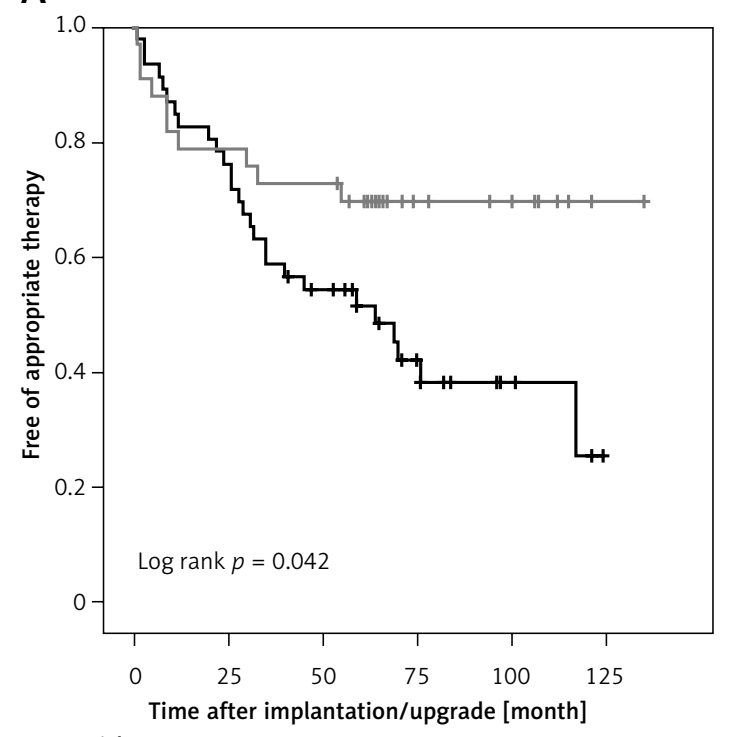

No. at risk
B

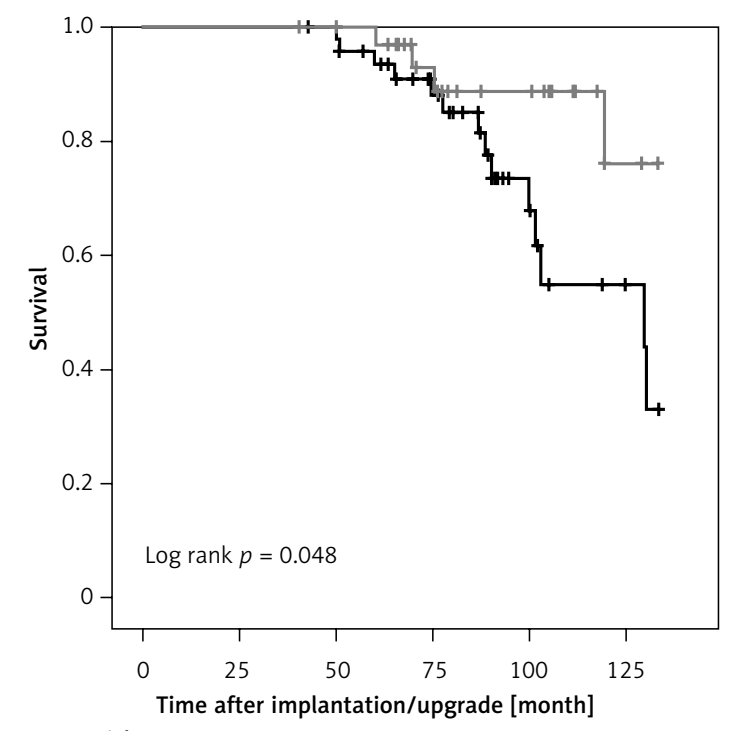

No. at risk

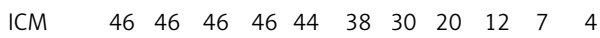

$\begin{array}{llllllllllll}\text { DCM } & 33 & 33 & 33 & 33 & 32 & 27 & 20 & 16 & 13 & 8 & 3\end{array}$

Figure 3. ICD therapy-free survival and cumulative survival in patients with CRT-D for primary prevention. A - Kaplan-Meier estimates for survival free of appropriate therapy (shock or anti-tachycardia pacing) delivered by CRT-D. B - Kaplan-Meier estimates for survival in CRT-D patients according to disease etiology

ICM - ischemic cardiomyopathy, DCM - non-ischemic dilated cardiomyopathy, CRT-D - cardiac resynchronization therapy combined with an implantable cardioverter defibrillator (ICD). 
Increased mortality and ICD therapies in ischemic versus non-ischemic dilated cardiomyopathy patients with cardiac resynchronization having survived until first device replacement

Table V. Univariate and multivariate Cox proportional hazard ratios (HR) for appropriate CRT-D intervention (shock or anti-tachycardia pacing) in patients with CRT-D implantation for primary prevention

\begin{tabular}{|c|c|c|c|c|}
\hline \multirow[t]{2}{*}{ Parameter } & \multicolumn{2}{|l|}{ Univariate } & \multicolumn{2}{|c|}{ Multivariate } \\
\hline & $\mathrm{HR}(95 \% \mathrm{Cl})$ & $P$-value & $\mathrm{HR}(95 \% \mathrm{Cl})$ & $P$-value \\
\hline Age, per 5 years & $1.102(0.917-1.324)$ & 0.303 & & \\
\hline Male & 3.781 (1.159-12.337) & 0.028 & $1.507(0.426-5.327)$ & 0.524 \\
\hline ICM & $2.088(1.009-4.319)$ & 0.047 & $2.500(1.142-5.476)$ & 0.022 \\
\hline Diabetes & $0.611(0.302-1.238)$ & 0.172 & & \\
\hline Hypertension & $1.048(0.506-2.167)$ & 0.900 & & \\
\hline $\mathrm{BMI}$, per $1 \mathrm{~kg} / \mathrm{m}^{2}$ & $0.969(0.905-1.038)$ & 0.374 & & \\
\hline Obesity & $0.595(0.276-1.280)$ & 0.184 & & \\
\hline Dyslipidemia & $1.420(0.736-2.742)$ & 0.296 & & \\
\hline Peripheral artery disease & $1.386(0.607-3.166)$ & 0.438 & & \\
\hline Previous stroke & $1.381(0.488-3.914)$ & 0.543 & & \\
\hline COPD & $1.773(0.836-3.763)$ & 0.136 & & \\
\hline Chronic renal failure & $0.954(0.496-1.834)$ & 0.887 & & \\
\hline Obstructive sleep apnea & $0.463(0.141-1.516)$ & 0.203 & & \\
\hline Central sleep apnea & $0.972(0.342-2.765)$ & 0.958 & & \\
\hline NSVT per year & $1.045(1.015-1.076)$ & 0.003 & $1.054(1.018-1.091)$ & 0.003 \\
\hline LVEF, per $5 \%$ & $0.692(0.547-0.875)$ & 0.002 & $0.685(0.529-0.886)$ & 0.004 \\
\hline
\end{tabular}

ICM - ischemic cardiomyopathy, BMI - body mass index, COPD - chronic obstructive pulmonary disease, NSVT - non-sustained ventricular tachycardia, LVEF - left ventricular ejection fraction.

Table VI. Univariate and multivariate Cox proportional hazard ratios (HR) for mortality in patients with CRT-D implantation for primary prevention

\begin{tabular}{|c|c|c|c|c|}
\hline \multirow[t]{2}{*}{ Parameter } & \multicolumn{2}{|l|}{ Univariate } & \multicolumn{2}{|c|}{ Multivariate } \\
\hline & $\mathrm{HR}(95 \% \mathrm{Cl})$ & $P$-value & HR $(95 \% \mathrm{Cl})$ & $P$-value \\
\hline Age, per 5 years & $1.277(0.945-1.725)$ & 0.112 & & \\
\hline Male & $4.634(0.616-34.874)$ & 0.136 & & \\
\hline ICM & $2.940(0.962-8.985)$ & 0.059 & & \\
\hline Diabetes & $0.751(0.266-2.116)$ & 0.588 & & \\
\hline Hypertension & $1.415(0.500-4.004)$ & 0.512 & & \\
\hline $\mathrm{BMI}$, per $1 \mathrm{~kg} / \mathrm{m}^{2}$ & $0.959(0.863-1.065)$ & 0.432 & & \\
\hline Obesity & $0.448(0.146-1.376)$ & 0.161 & & \\
\hline Dyslipidemia & $1.640(0.645-4.169)$ & 0.299 & & \\
\hline Peripheral artery disease & 3.645 (1.341-9.910) & 0.011 & $2.871(1.028-8.015)$ & 0.044 \\
\hline Previous stroke & $1.226(0.281-5.358)$ & 0.786 & & \\
\hline COPD & $4.072(1.599-10.371)$ & 0.003 & $3.412(1.319-8.829)$ & 0.011 \\
\hline Chronic renal failure & $2.439(0.943-6.310)$ & 0.066 & & \\
\hline Obstructive sleep apnea & $0.520(0.067-4.018)$ & 0.531 & & \\
\hline Central sleep apnea & $3.267(1.145-9.321)$ & 0.027 & $1.949(0.549-6.920)$ & 0.302 \\
\hline NSVT per year & $0.978(0.909-1.052)$ & 0.553 & & \\
\hline LVEF, per $5 \%$ & $0.856(0.624-1.173)$ & 0.332 & & \\
\hline
\end{tabular}

ICM - ischemic cardiomyopathy, BMI - body mass index, COPD - chronic obstructive pulmonary disease, NSVT - non-sustained ventricular tachycardia, LVEF - left ventricular ejection fraction. 


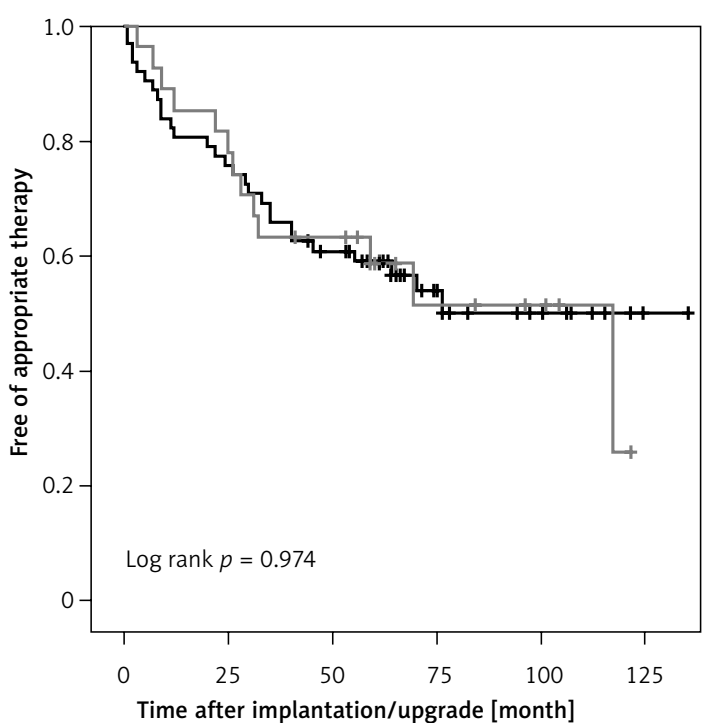

No. at risk

$\begin{array}{llllllllllll}\text { Direct } & 63 & 50 & 47 & 40 & 32 & 23 & 15 & 10 & 6 & 3 & 1\end{array}$

Upgrade $\begin{array}{lllllllllll}25 & 22 & 21 & 16 & 12 & 8 & 5 & 4 & 3 & 2 & 0\end{array}$

$\curvearrowleft$ Direct implantation $\square$ Upgrade

Figure 4. ICD therapy-free survival in directly implanted versus upgraded CRT-D patients. Kaplan-Meier estimates for survival free of appropriate therapy (shock or anti-tachycardia pacing) delivered by CRT-D in patients with direct implantation of CRT-D or upgrade from previously implanted ICD, pacemaker or CRT-P

$I C D$ - implantable cardioverter defibrillator, CRT-D cardiac resynchronization therapy combined with an ICD, CRT-P-CRT pacemaker.

failure with reduced LVEF and therefore trigger the implantation of CRT-D or ICD devices.

During the mean follow-up of 76 months, 41 (46.6\%) patients had any device therapy (57.7\% of ICM patients, $30.6 \%$ of DCM patients). Kaplan-Meier analysis revealed a significantly higher rate of any ICD therapy in ICM patients. This was mainly driven by a higher rate of ATP. To date, most studies have reported similar VT/ VF or shock rates in CRT-D patients when comparing ICM and DCM $[6,9,11]$. In a post-hoc analysis of the MADIT-CRT study population Kutyifa et al. reported an incidence of first VT/VF of $27 \%$ in ICD and $22 \%$ in CRT-D patients after a follow-up of 40 months [13]. In our study, we observed at 40 months a higher rate of $38.7 \%$ (therapy for VT/ VF). In another study including more than 67,000 patients the cumulative shock incidence was 33\% (ICD) and 28\% (CRT-D) 5 years after device implantation [14]. This is very similar to our shock incidence of $27.5 \%$ at 60 months of follow-up. Valles et al. reported an annual shock rate in ICD patients of $10 \%$ for ICM and $4 \%$ for DCM [15]. Taken together, some previous studies observed at least numerically higher shock rates in ICM patients when compared to DCM patients, but their follow-up duration rarely exceeded 40 months and mainly ICD shocks were analyzed. Our study now adds more than 3 years of extra longitudinal observation and additional data on ATP. Therefore, extended follow-up and the combination of shock and ATP as the endpoint might explain why the presented significant differences between ICM and DCM patients were detected now. Both patient populations have different patterns of myocardial scarring with predominantly endocardial scar formation following ischemia versus more isolated mid-myocardial or epicardial scars in DCM patients. For both etiologies it was shown before that myocardial scarring has an impact on the occurrence of arrhythmia [16, 17]. Therefore, the differing patterns of scar formation might translate into differential arrhythmia and ICD therapy rates.

In addition to the higher rate of ICD interventions, we could also demonstrate a significant in crease in mortality for patients suffering from ICM compared to DCM patients. So far there have been conflicting data on mortality when comparing both cardiomyopathies treated with CRT-D, ranging from a reduction in all-cause mortality in ICM patients [4], through no difference in mortality [6], to a survival benefit in DCM patients [9]. The recently published DANISH-Trial reported no reduction in mortality following prophylactic implantation of an ICD in heart failure patients without coronary artery disease [18]. Interestingly, more than $50 \%$ of the study population were treated with CRT-D or CRT-P devices. Here we report a higher risk for ICD interventions and increased mortality in ICM patients, likewise in the subgroup of primary prophylactic implanted devices. The excess mortality seems to be at least in part due to a higher rate of co-morbidities in ICM patients. Peripheral artery disease, COPD and chronic renal failure share pathophysiological mechanisms as well as risk factors with coronary artery disease, and rates were numerically higher in patients with ICM. Due to our small patient population these differences were not significant, but multivariate analysis identified these three parameters as strong predictors of mortality. This is in line with previous studies reporting increased mortality in patients with coronary artery disease combined with COPD [19-21], peripheral artery disease [22, 23] or chronic renal failure [24].

Interestingly, univariate analysis for mortality prediction revealed a significant impact of central but not obstructive sleep apnea, which is in line with a recent meta-analysis by Nakamura et al. [25]. Most certainly due to the small patient population with central sleep apnea, no significant predictive value was observed in the multivariate analysis.

We could identify LVEF, NSVT per year and ICM as independent predictors of ICD intervention. For MADIT-CRT patients it has previously been shown that each $5 \%$ of LVEF was associated with a $30 \%$ 
reduction in risk for fast VT/VF [13]. In our study we could reproduce this amount of risk reduction $(H R=0.703)$. For ICD patients it has been demonstrated before that the occurrence of NSVT is accompanied by an increased risk of ICD interventions [26, 27], which we could now also demonstrate in CRT-D patients (6\% increase in risk for ICD intervention per occurrence of NSVT per year).

We did not observe any difference in eventfree survival between upgraded and directly implanted CRT-D devices. Since significantly more ICM patients were upgraded and patients with ICM showed higher rates of device therapies, this might have influenced the results. Therefore, we analyzed ICM and DCM patients separately and event-free survival remained similar between upgrade and direct implantation.

In addition to the small size of our study population, the retrospective design implies several known limitations. Differences in CRT-D programming for device therapies over time might have influenced the rate of shocks and ATP [12], but similarly in patients with ICM and DCM. We report real-world data from a typical non-selected patient population during standard clinical practice. Throughout the whole study period, the devices were programmed according to current recommendations at the respective point in time. Furthermore, our pre-selection to include only patients who survived until the first CRT-D replacement due to end of the battery lifespan might have induced a certain bias, potentially compromising translation of our results to all patients immediately after CRT-D implantation.

In conclusion, this present study reveals a different rate of arrhythmic events, subsequent ICD therapies and mortality depending on the underlying etiology of the cardiomyopathy. Patients with ICM had significantly more device interventions during long-term follow-up, an overall $150 \%$ higher risk for shock/ATP application and increased mortality. The LVEF prior to device implantation, ICM as heart failure etiology and NSVT were the strongest predictors of device therapy, whereas peripheral artery disease, COPD and chronic renal failure were the strongest mortality predictors.

\section{Acknowledgments}

Thomas Beiert and Swanda Straesser contributed equally to this work.

\section{Conflict of interest}

The authors declare no conflict of interest.

\section{References}

1. Brignole M, Auricchio A, Baron-Esquivias G, et al. 2013 ESC Guidelines on cardiac pacing and cardiac resyn- chronization therapy: the Task Force on cardiac pacing and resynchronization therapy of the European Society of Cardiology (ESC). Developed in collaboration with the European Heart Rhythm Association (EHRA). Eur Heart J 2013; 34: 2281-329.

2. Priori SG, Blomstrom-Lundqvist C, Mazzanti A, et al. 2015 ESC Guidelines for the management of patients with ventricular arrhythmias and the prevention of sudden cardiac death: The Task Force for the Management of Patients with Ventricular Arrhythmias and the Prevention of Sudden Cardiac Death of the European Society of Cardiology (ESC). Endorsed by: Association for European Paediatric and Congenital Cardiology (AEPC). Eur Heart J 2015; 36: 2793-867.

3. Bardy GH, Lee KL, Mark DB, et al. Amiodarone or an implantable cardioverter-defibrillator for congestive heart failure. N Engl I Med 2005; 352: 225-37.

4. Barra S, Providencia R, Tang A, Heck P, Virdee M, Agarwal S. Importance of implantable cardioverter-defibrillator back-up in cardiac resynchronization therapy recipients: a systematic review and meta-analysis. J Am Heart Assoc 2015; 4: pii: e002539.

5. Saini A, Kannabhiran M, Reddy P, Gopinathannair R, Olshansky B, Dominic P. Cardiac resynchronization therapy may be antiarrhythmic particularly in responders. JACC Clin Electrophysiol 2016; 2: 307-16.

6. Wasmer K, Kobe J, Andresen D, et al. Comparing outcome of patients with coronary artery disease and dilated cardiomyopathy in ICD and CRT recipients: data from the German DEVICE-registry. Clin Res Cardiol 2013; 102: 513-21.

7. Barsheshet A, Goldenberg I, Moss AJ, et al. Response to preventive cardiac resynchronization therapy in patients with ischaemic and nonischaemic cardiomyopathy in MADIT-CRT. Eur Heart J 2011; 32: 1622-30.

8. Moss AJ, Hall WJ, Cannom DS, et al. Cardiac-resynchronization therapy for the prevention of heart-failure events. N Engl J Med 2009; 361: 1329-38.

9. McLeod CJ, Shen W, Rea RF, et al. Differential outcome of cardiac resynchronization therapy in ischemic cardiomyopathy and idiopathic dilated cardiomyopathy. Heart Rhythm 2011; 8: 377-82.

10. Smith T, Theuns, Dominic AMJ, Caliskan K, Jordaens L. Long-term follow-up of prophylactic implantable cardioverter-defibrillator-only therapy: comparison of ischemic and nonischemic heart disease. Clin Cardiol 2011; 34: 761-7.

11. Verhagen MP, van Boven N, Ruiter JH, Kimman GP, Tahapary GJ, Umans VA. Follow-up of implantable cardioverter-defibrillator therapy: comparison of coronary artery disease and dilated cardiomyopathy. Neth Heart J 2014; 22: 431-7.

12. Moss AJ, Schuger C, Beck CA, et al. Reduction in inappropriate therapy and mortality through ICD programming. N Engl J Med 2012; 367: 2275-83.

13. Kutyifa V, Moss AJ, Solomon SD, et al. Reduced risk of life-threatening ventricular tachyarrhythmias with cardiac resynchronization therapy: relationship to left ventricular ejection fraction. Eur J Heart Fail 2015; 17: 971-8.

14. Saba S, Adelstein E, Wold N, Stein K, Jones P. Influence of patients' age at implantation on mortality and defibrillator shocks. Europace 2017; 19: 802-7.

15. Valles AG, Khawaja FJ, Gersh BJ, et al. Implantable cardioverter defibrillators in patients with valvular cardiomyopathy. J Cardiovasc Electrophysiol 2012; 23: 1326-32. 
16. Kwon DH, Halley CM, Carrigan TP, et al. Extent of left ventricular scar predicts outcomes in ischemic cardiomyopathy patients with significantly reduced systolic function: a delayed hyperenhancement cardiac magnetic resonance study. JACC Cardiovasc Imaging 2009; 2: 34-44.

17. Neilan TG, Coelho-Filho OR, Danik SB, et al. CMR quantification of myocardial scar provides additive prognostic information in nonischemic cardiomyopathy. JACC Cardiovasc Imaging 2013; 6: 944-54.

18. Kober L, Thune JJ, Nielsen JC, et al. Defibrillator implantation in patients with nonischemic systolic heart failure. N Engl J Med 2016; 375: 1221-30.

19. Andell P, Koul S, Martinsson A, et al. Impact of chronic obstructive pulmonary disease on morbidity and mortality after myocardial infarction. Open Heart 2014; 1 : e000002.

20. Boschetto P, Beghe B, Fabbri LM, Ceconi C. Link between chronic obstructive pulmonary disease and coronary artery disease: implication for clinical practice. Respirology 2012; 17: 422-31.

21. Zhang M, Cheng Y, Zheng W, et al. Impact of chronic obstructive pulmonary disease on long-term outcome in patients with coronary artery disease undergoing percutaneous coronary intervention. Biomed Res Int 2016; 2016: 8212459.

22. Subherwal S, Patel MR, Kober L, et al. Peripheral artery disease is a coronary heart disease risk equivalent among both men and women: results from a nationwide study. Eur J Prev Cardiol 2015; 22: 317-25.

23. Grenon SM, Vittinghoff E, Owens CD, Conte MS, Whooley $\mathrm{M}$, Cohen BE. Peripheral artery disease and risk of cardiovascular events in patients with coronary artery disease: insights from the Heart and Soul Study. Vasc Med 2013; 18: 176-84.

24. Cai Q, Mukku VK, Ahmad M. Coronary artery disease in patients with chronic kidney disease: a clinical update. Curr Cardiol Rev 2013; 9: 331-9.

25. Nakamura S, Asai K, Kubota Y, et al. Impact of sleep-disordered breathing and efficacy of positive airway pressure on mortality in patients with chronic heart failure and sleep-disordered breathing: a meta-analysis. Clin Res Cardiol 2015; 104: 208-16.

26. Verma A, Sarak B, Kaplan AJ, et al. Predictors of appropriate implantable cardioverter defibrillator (ICD) therapy in primary prevention patients with ischemic and nonischemic cardiomyopathy. Pacing Clin Electrophysio 2010; 33: 320-9.

27. Chen J, Johnson G, Hellkamp AS, et al. Rapid-rate nonsustained ventricular tachycardia found on implantable cardioverter-defibrillator interrogation: relationship to outcomes in the SCD-HeFT (Sudden Cardiac Death in Heart Failure Trial). J Am Coll Cardiol 2013; 61: 2161-8. 